Pacific Journal of Mathematic 


\title{
ON THE NUMBER OF DISSIMILAR LINE-SUBGRAPHS OF A GIVEN GRAPH
}

\author{
Frank HARARY
}

1. Introduction. Two enumeration formulas are obtained in this paper. The first provides a solution for the counting polynomial which gives the number of dissimilar line-subgraphs of a given graph, and is a generalization of the formula due to Pólya for the number of graphs which appears in [2]. The second serves to find the number of graphs in which a prescribed subgraph is distinguished or rooted. The special case in which a single point of the graph is distinguished is called a rooted graph [3, p. 76]. The number of rooted graphs also appears in [2].

Both of these results utilize in an essential way the classical enumeration theorem of Pólya [4] which enables one to express the configuration-counting series in terms of the configuration group and the figure-counting series. In order that these results be self-contained, we briefly review Pólya's Theorem, specialized to one variable. Let figure be an undefined term. To each figure there is assigned a nonnegative integer called its content. Let $\varphi_{k}$ denote the number of different figures of content $k$. The figure-counting series $\varphi(x)$ is defined by

$$
\varphi(x)=\sum_{k=0}^{\infty} \varphi_{k} x^{k} .
$$

Let $\Gamma$ be a permutation group of degree $s$ and order $h$. A configuration of length $s$ is a sequence of $s$ figures. The content of a configuration is the sum of the contents of its figures. Two configurations are $\Gamma$-equivalent, if there is a permutation of $\Gamma$ sending one into the other. Let $F_{k}$ denote the number of $\Gamma$-inequivalent figures of content $k$. The configuration-counting series $F(x)$ is defined by

$$
F(x)=\sum_{k=0}^{\infty} F_{k} x^{k}
$$

The permutation group $\Gamma$ will be called the configuration group.

Pólya's Theorem expresses $F(x)$ in terms of $\varphi(x)$ and $\Gamma$, using the cycle index of $\Gamma$. Let $h_{j_{1} j_{2} \ldots j_{s}}$ be the number of elements of $\Gamma$ of type $\left(j_{1}, j_{2}, \cdots, j_{s}\right)$, that is, having $j_{k}$ cycles of length $k,(k=1, \cdots, s)$ so that

Received September 20, 1954. This work was supported by a grant from the Rockefeller Foundation to the Research Center for Group Dynamics, University of Michigan. 


$$
1 \cdot j_{1}+2 \cdot j_{2}+\cdots+s \cdot j_{s}=s .
$$

Let $f_{1}, f_{2}, \cdots, f_{s}$ be indeterminates. Then $Z(\Gamma)$, the cycle index of $\Gamma$, is defined as in $[4, p .159]$ by

$$
Z(\Gamma)=\frac{1}{h} \sum_{(j)} h_{j_{1} j_{2} \cdots j_{s}} f_{1}^{j_{1}} f_{2}^{j_{2}} \cdots f_{s}^{j_{s}},
$$

where the sum is taken over all partitions $(j)$ of $s$ satisfying (1). For any series $f(x)$, let $Z(\Gamma, f(x))$ be the series obtained from $Z(\Gamma)$ on replacing each indeterminate $f_{k}$ by $f\left(x^{k}\right)$.

Polya's TheOREM [4, p. 163]. The configuration-counting series is obtained when the figure-counting series is substituted into the cycle index of the configuration group

$$
F(x)=Z(\Gamma, \varphi(x))
$$

2. Enumeration of the dissimilar line-subgraphs of a given graph.

A graph $\mathscr{S}$ consists of a finite set II of points $A_{1}, A_{2}, \cdots, A_{p}$ together with a given subset $A$ of the collection of all unordered pairs of distinct points. The elements of $A$ are called the lines of $\mathscr{G}$. Two points of $\mathscr{C}$ are adjacent if there is a line of $\mathscr{C}$ containing them. Two graphs are isomorphic if there is a one-to-one correspondence between their point sets which preserves adjacency. An automorphism of a graph $\mathscr{C}$ is an isomorphism of $\mathscr{C}$ with itself. It is well known that the collection of all automorphisms of $\mathscr{C}$ constitutes a group [3, p. 5]. Let $\Gamma_{0}(\mathscr{S})$ be the group of the graph $\mathscr{C}$. Then $\Gamma_{0}(\mathscr{S})$ is a permutation group of degree $p=$ the number of points of $\mathscr{C}$. We shall call $\Gamma_{0}(\mathscr{S})$ the point-group of $\mathscr{G}$.

A line-subgraph or briefly $\ell$-subgraph of $\mathscr{C}$ is a graph whose point set is that of $\mathscr{C}$ and whose line set is a subset of the line set of $\mathscr{S}$. Two points $A, B$ of $\mathscr{S}$ are similar if there is an automorphism of $\mathscr{S}$ mapping $A$ onto $B$. Two lines $A B, C D$ of $\mathscr{C}$ are similar if there is an automorphism of $\mathscr{P}$ mapping $\{A, B\}$ onto $\{C, D\}$. Two $\ell$-subgraphs of $\mathscr{G}$ are similar if there is an automorphism of the graph (S) which maps the lines of one onto the lines of the other. Similarity being an equivalence relation, the number of dissimilar $\ell$-subgraphs of $\mathscr{C}$ refers to the number of similarity classes of $\ell$-subgraphs.

The problem solved by Theorem 1 is the following. Given an arbitrary graph $\mathscr{G}$ with $p$ points and $q$ lines, how many dissimilar $\ell$-subgraphs does $\mathscr{S}$ have containing $k$ lines, $k=0,1, \cdots, q$ ? Using the terminology of Pólya's Theorem, the configurations to be counted 
are the $t$-subgraphs of $\mathscr{S}$ which are inequivalent with respect to the automorphisms of $\mathscr{G}$. The figures out of which these configurations are built are the pairs of distinct points of $\mathscr{C}$. The content of a figure is 0 if the two points are non-adjacent and is 1 if they are adjacent. Hence the figure-counting series is $\varphi(x)=1+x$.

The content of an $\ell$-subgraph is the number $k$ of lines it contains. With the basic graph $\mathscr{G}$ fixed throughout this discussion, let $g_{k}$ be the number of dissimilar $\ell$-subgraphs having $k$ lines. Then the configuration-counting series or counting polynomial $g(x)$ is given by

$$
g(x)=\sum_{k=0}^{q} g_{k} x^{k}
$$

In order to apply Pólya's Theorem to express $g(x)$, it remains to find the configuration group.

We require the permutation group $\Gamma$ of degree $q$ which acts on the lines of $\mathscr{C}$, having the property that two $\ell$-subgraphs are similar if and only if they are $\Gamma$-equivalent. Clearly this group consists of all permutations of the set $A$ induced by the automorphisms of $\mathscr{C}$, that is, by the elements of $\Gamma_{0}(\mathscr{S})$. Let $\Gamma_{1}(\mathscr{S})$, the line-group of $\mathscr{C}$, denote this group; then $\Gamma=\Gamma_{1}(\mathscr{S})$, a permutation group of degree $q$. Collecting these results and applying Pólya's Theorem, we have the following theorem.

THEOREM 1. The counting polynomial for the number of dissimilar $\ell$-subgraphs of $\mathscr{G}$ is obtained by substituting $1+x$ into the cycle index of the line-group of $\mathscr{B}$

$$
g(x)=\sum_{k=0}^{q} g_{k} x^{k}=Z\left(\Gamma_{1}(\mathscr{Y}), 1+x\right) .
$$

This specializes at once to the counting polynomial $g_{p}(x)$ of [2] for the number of different graphs of $p$ points when one takes for $\mathscr{G}$ the complete graph of $p$ points, that is, the graph in which any two distinct points are adjacent. For two graphs are different if they are not isomorphic, which means that they are dissimilar $/$-subgraphs of the complete $p$ point graph.

We next apply Theorem 1 to all the graphs of 4 points. These are drawn in Figure 1, in which $\mathscr{O}_{k n}$ denotes the $n$th graph of 4 points and $k$ lines. In order to describe the line-groups of these graphs concisely, the following notations are employed. Let $E_{n}, S_{n}, D_{n}$ be the identity, symmetric, and dihedral groups of degree $n$ respectively. Let $G_{1} \times G_{2}$ denote the direct product of the permutation groups $G_{1}$ and $G_{2}$ which act on two disjoint sets of objects. Let $K_{4}$ (the Klein four-group) 


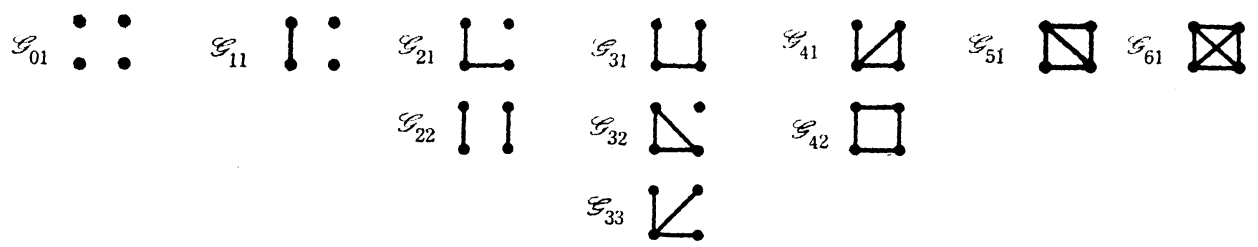

Figure 1

denote the permutation group whose elements are

$$
(a)(b)(c)(d),(a b)(c d),(a d)(b c),(a c)(b d) .
$$

Finally, let $P\left(S_{n}\right)$ be the pair group of $S_{n}$, that is, the group of degree $n(n-1) / 2$ which is abstractly isomorphic with $S_{n}$, but acts on the pairs of the $n$ objects permuted by $S_{n}$.

In Table 1 below, each graph $\mathscr{G}$ of 4 points is indicated in the first column, its line-group $\Gamma_{1}(\mathscr{S})$ in the second column, the cycle index $Z\left(\Gamma_{1}(\mathscr{S})\right)$ in the third column, and the counting polynomial $g(x)$ $=Z\left(\Gamma_{1}(\mathscr{G}), 1+x\right)$ in the last column.

Table 1

\begin{tabular}{cccl}
$\mathcal{I}$ & $\Gamma_{1}(\mathcal{G})$ & $Z\left(\Gamma_{1}(\mathcal{G})\right)$ & \multicolumn{1}{c}{$g(x)=Z\left(\Gamma_{1}(\mathcal{G}), 1+x\right)$} \\
\hline $\mathcal{G}_{31}$ & $1=E_{0}$ & 1 & 1 \\
$\mathcal{G}_{11}$ & $E=E_{1}$ & $f_{1}$ & $1+x$ \\
$\mathcal{G}_{21}$ & $S_{2}$ & $\left(f_{1}^{2}+f_{2}\right) / 2$ & $1+x+x^{2}$ \\
$\mathcal{G}_{22}$ & $S_{2}$ & $\left(f_{1}^{2}+f_{2}\right) / 2$ & $1+x+x^{2}$ \\
$\mathcal{G}_{31}$ & $S_{2} \times E$ & $\left(f_{1}^{3}+f_{1} f_{2}\right) / 2$ & $1+2 x+2 x^{2}+x^{3}$ \\
$\mathcal{G}_{32}$ & $S_{3}$ & $\left(f_{1}^{3}+3 f_{1} f_{2}+2 f_{3}\right) / 6$ & $1+x+x^{2}+x^{3}$ \\
$\mathcal{G}_{33}$ & $S_{3}$ & $\left(f_{1}^{3}+3 f_{1} f_{2}+2 f_{3}\right) / 6$ & $1+x+x^{2}+x^{3}$ \\
$\mathcal{G}_{41}$ & $S_{2} \times E_{2}$ & $\left(f_{1}^{4}+f_{1}^{2} f_{2}\right) / 2$ & $1+3 x+4 x^{2}+3 x^{3}+x^{4}$ \\
$\mathcal{G}_{42}$ & $D_{4}$ & $\left(f_{1}^{4}+3 f_{2}{ }^{2}+2 f_{1}^{2} f_{2}+2 f_{4}\right) / 8$ & $1+x+2 x^{2}+x^{3}+x^{4}$ \\
$\mathcal{G}_{51}$ & $K_{4} \times E$ & $\left(f_{1}{ }^{5}+3 f_{1} f_{2}{ }^{2}\right) / 4$ & $1+2 x+4 x^{2}+4 x^{3}+2 x^{4}+x^{5}$ \\
$\mathcal{G}_{61}$ & $P\left(S_{4}\right)$ & $\left(f_{1}{ }^{6}+9 f_{1}{ }^{2} f_{2}{ }^{2}+8 f_{3}{ }^{2}+6 f_{2} f_{4}\right) / 24$ & $1+x+2 x^{2}+3 x^{3}+2 x^{4}+x^{5}+x^{6}$
\end{tabular}

We conclude this section with Figure 2 which illustrates the dissimilar $\ell$-subgraphs for the case $\mathscr{C}=\mathscr{V}_{51}$.

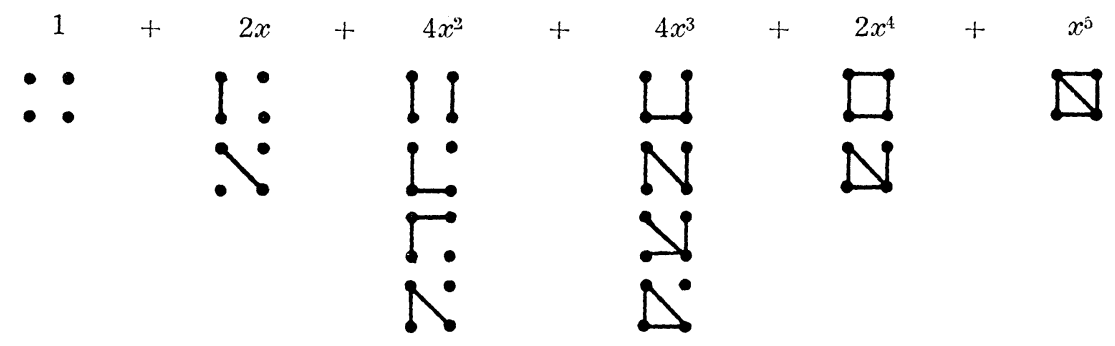

Figure 2 
3. Enumeration of Grooted graphs. A rooted graph is one in which one of the points is singled out from the others. The distinguished point is called the root $R$. Two rooted graphs $\mathscr{G}_{1}, \mathscr{G}_{2}$ with roots $R_{1}, R_{2}$ are isomorphic if there exists an isomorphism of $\mathscr{G}_{1}$ with $\mathscr{G}_{2}$ considered as ordinary unrooted graphs, which maps $R_{1}$ onto $R_{2}$.

In this section the notion of rooted gragh will be generalized. Two graphs having the same point set $\Pi$ as $\mathscr{C}$ will be isomorphic as $\mathscr{G}$ rooted graphs, speaking loosely, if they are similar when viewed from $\mathscr{G}$. A $\mathscr{G}$-rooted graph $H$ is one, defined on the same point set as $\mathscr{G}$, in which the graph $\mathscr{G}$ is distinguished. It is not necessary that $\mathscr{G}$ be an $\ell$-subgraph of $H$ (as will be seen in Figure 3). The counting polynomial whose coefficients give the number of such $\mathscr{G}$-rooted graphs of $p$ points and $k$ lines for $k=0, \cdots, p(p-1) / 2$ is the result of Theorem 2.

Let the arbitrary graph $\mathscr{G}$ with $p$ points and $q$ lines be fixed throughout this discussion. Two graphs $\mathscr{G}_{1}, \mathscr{G}_{2}$, both having the same point set $\Pi$ as $\mathscr{G}$, are isomorphic as $\mathscr{G}$-rooted graphs if there is an isomorphic mapping of $\mathscr{G}_{1}$ with $\mathscr{G}_{2}$ which is also an automorphism of $\mathscr{G}$. Let $h_{k}$ be the number of different (that is, non-isomorphic) $\mathscr{G}$ rooted graphs of $k$ lines and let

$$
h(x)=\sum_{k=0}^{p(p-1) / 2} h_{k} x^{k}
$$

As for /-subgraphs, a $\mathscr{C}$-rooted graph is a configuration whose figures are the pairs of distinct points of $\mathscr{G}$, and the figure-counting series is again $1+x$. It follows from the definition of isomorphism of two $\mathscr{G}$ rooted graphs that the configuration group $\Gamma$ is the pair group of the point-group of $\mathscr{G}$. We define the pair group $P(H)$ of a permutation group $H$ of degree $s$ as the permutation group of degree $s(s-1) / 2$ which acts on the set of all pairs of distinct elements of the object set of $H$, each of the elements of $P(H)$ being induced by an element of $H$. Thus $P(H)$ and $H$ are isomorphic as abstract groups.

We have seen that for $\mathscr{G}$-rooted graphs, the figure series is $1+x$ and the configuration group $\Gamma=P\left(\Gamma_{0}(\mathscr{G})\right)$. We now apply Pólya's Theorem.

THEOREM 2. The counting polynomial for G-rooted graphs is obtained when one substitutes $1+x$ into the cycle index of the pair group of the point-group of $\mathscr{G}$ :

$$
h(x)=\sum_{k=0}^{p(p-1) / 2} h_{k} x^{k}=Z\left(P\left(\Gamma_{0}(\mathscr{G})\right), 1+x\right) .
$$


It is interesting that this formula is a generalization of both the number of ordinary graphs and the number of rooted graphs [2]. For the choice of $\mathscr{G}$ as the complete graph of $p$ points, we have $P\left(\Gamma_{0}(\mathscr{G})\right.$ ) $=\Gamma_{1}(\mathscr{G})$, so that, as remarked after Theorem $1, h(x)=g_{p}(x)=$ the counting polynomial for all $p$ point graphs. On the other hand, if $\mathscr{G}$ is taken as a graph with two components, one a single isolated point and the other a complete graph of $p-1$ points, then

$$
h(x)=Z\left(P\left(\Gamma_{0}(\mathscr{C})\right), 1+x\right)=Z\left(P\left(S_{p-1} \times E\right), 1+x\right)
$$

which is exactly the formula in [2] for $G_{p}(x)$, the counting polynomial for the rooted graphs of $p$ points.

After writing $Z\left(\Gamma_{0}(\mathscr{G})\right)$, the expression for $Z\left(P\left(\Gamma_{0}(\mathscr{G})\right)\right)$ is obtained readily using a formula from [2], which appears as equation (8) below.

For convenience, let $\Gamma$ be any permutation group and

$$
Z_{2}(\Gamma)=Z(P(\Gamma)) \text {. }
$$

Then, referring to (2),

$$
Z_{2}(\Gamma)=\frac{1}{h} \sum_{(j)} h_{j_{1} j_{2} \cdots j_{s}} Z_{2}\left(f_{1}^{j_{1}} f_{2}^{j_{2}} \cdots f_{s}^{j_{s}}\right)
$$

where, from [2], one has

$$
\begin{gathered}
Z_{2}\left(f_{1}^{j_{1}} f_{2}^{j_{2}} \cdots f_{s s}^{j}\right)=\sum_{n=0}^{[(s-1) / 2]} f_{2 n+1}^{n j_{2 n+1}+(2 n+1)}\left({ }_{2}^{j} j_{2 n+1}\right) \cdot \sum_{n=1}^{[s / 2]}\left(f_{n} f_{2 n}^{n-1}\right)^{j_{2 n} n} f_{2 n}^{2 n}\left({ }_{2}^{j_{2 n}}\right) \\
\cdot \sum_{1 \leqq q<r \leqq s} f_{m}^{j_{q}^{j} r_{r}^{\alpha(q, r)}},
\end{gathered}
$$

in which $m(q, r)$ and $d(q, r)$ denote least common multiple and greatest common divisor respectively. Combining (7) and (8) with (2), one has a formula for $Z_{2}\left(\Gamma_{0}(\mathscr{G})\right)$ once $Z\left(\Gamma_{0}(\mathscr{G})\right)$ is known.

We conclude by applying Theorem 2 to the 4 point graphs given in Figure 1. Let $\mathscr{C}^{\prime}$ denote the complement of $\mathscr{G}$, that is, the graph whose point set coincides with that of $\mathscr{G}$, in which two points are adjacent if and only if they are not adjacent in $\mathscr{G}$. Since $\Gamma_{0}\left(\mathscr{G}^{\prime}\right)=$ $\Gamma_{0}(\mathscr{S})$, we see that the number of $\mathscr{S}$-rooted graphs equals the number of $\mathscr{C}^{\prime}$-rooted graphs. Hence it is appropriate to divide the graphs of Figure 1 into pairs $\mathscr{C}, \mathscr{G}^{\prime}$. Note that $\mathscr{G}_{31}$ and its complement are isomorphic, but otherwise each of these graph pairs contains distinct graphs. The results for $h(x)$ are given in Table 2.

We illustrate the process of calculating the polynomials in Table 2 with $\mathscr{C}_{22}$ or $\mathscr{G}_{22}$. 
Table 2

\begin{tabular}{cccc}
\multicolumn{2}{c|}{$\mathcal{G}$} & $h(x)$ \\
\hline $\mathcal{G}_{01}$ & or & $\mathcal{G}_{61}$ & $g_{4}(x)=1+x+2 x^{2}+3 x^{3}+2 x^{4}+x^{5}+x^{6}$ \\
$\mathcal{G}_{11}$ & or & $\mathcal{G}_{51}$ & $1+3 x+6 x^{2}+3 x^{3}+6 x^{4}+3 x^{5}+x^{6}$ \\
$\mathcal{G}_{21}$ & or & $\mathcal{G}_{41}$ & $1+4 x+9 x^{2}+12 x^{3}+9 x^{4}+4 x^{5}+x^{6}$ \\
$\mathcal{G}_{22}$ & or & $\mathcal{G}_{42}$ & $1+2 x+4 x^{2}+5 x^{3}+4 x^{4}+2 x^{5}+x^{6}$ \\
& $\mathcal{G}_{31}$ & $1+4 x+9 x^{2}+12 x^{3}+9 x^{4}+4 x^{5}+x^{6}$ \\
$\mathcal{G}_{32}$ & or & $\mathcal{G}_{33}$ & $G_{4}(x)=1+2 x+4 x^{2}+6 x^{3}+4 x^{4}+2 x^{5}+x^{6}$ \\
& $\Gamma_{0}\left(\mathscr{G}_{42}\right)=$ & $\Gamma_{0}\left(\mathscr{S}_{22}\right)=D_{4}$ \\
& $Z\left(D_{4}\right)=\frac{1}{8}\left(f_{1}^{4}+3 f_{2}^{2}+2 f_{1}^{2} f_{2}+2 f_{4}\right)$.
\end{tabular}

Applying (8), one gets

$$
Z_{2}\left(D_{4}\right)=\frac{1}{8}\left(f_{1}^{6}+5 f_{1}^{2} f_{2}^{2}+2 f_{2} f_{4}\right),
$$

so that the resulting polynomial is

$$
h(x)=1+2 x+4 x^{2}+5 x^{3}+4 x^{4}+2 x^{5}+x^{6} .
$$

The coefficients in this polynomial count the $\mathscr{C}$-rooted graphs of Figure 3. We first redraw the diagrams of graphs $\mathscr{G}_{22}$ and $\mathscr{G}_{42}$ so that they appear as complements on the same point set.

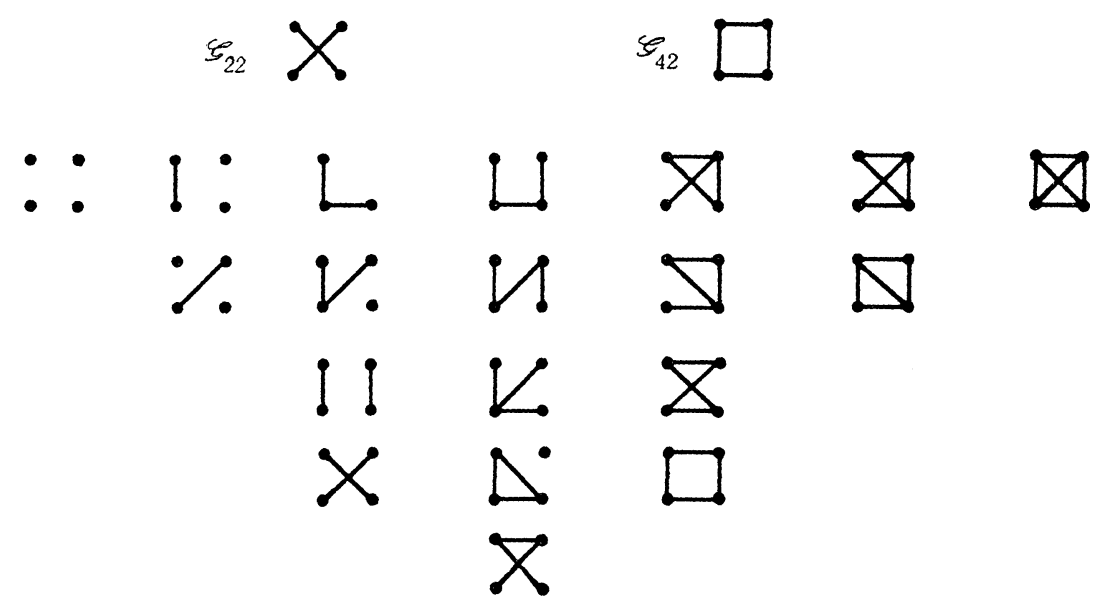

Figure 3

Finally we observe that both of these counting problems have counterparts in the theory of binary relations [1]. They serve to enumerate dissimilar symmetric subrelations over the same field as a given symmetric irreflexive relation and the " $\mathscr{R}$-rooted" symmetric irreflexive 
relation $\mathscr{R}$. The extensions to relations which are not necessarily symmetric or irreflexive correspond to the analogous counting problems for directed graphs and for graphs in the wider sense in which loops or slings $^{1}[3$, p. 3] may occur, and offer no difficulty.

\section{REFERENCES}

1. R.L. Davis, The number of structures of finite relations, Proc. Amer. Math. Soc., 4 (1953), 486-495.

2. F. Harary, The number of linear, directed, rooted, and connected graphs, Trans. Amer. Math. Soc., 78 (1955), 445-463.

3. D. König, Theorie der endlichen und unendlichen Graphen, Leipzig 1936; reprinted New York, 1950.

4. G. Pólya, Kombinatorische Anzahlbestimmungen für Gruppen, Graphen, und chemische Verbindungen, Acta Math., 68 (1937), 145-254.

UNIVERSITY OF MICHIGAN

1 A sling is a line joining a point with itself. 


\section{PACIFIC JOURNAL OF MATHEMATICS}

\section{EDITORS}

H. L. Royden

Stanford University

Stanford, California

E. HewitT

University of Washington

Seattle 5 , Washington
R. P. Dilworth

California Institute of Technology Pasadena 4, California

E. G. Straus

University of California

Los Angeles 24, California

\section{ASSOCIATE EDITORS}

E. F. BECKENBACH

C. E. BURGESS

H. BUSEMANN

H. FEDERER

\author{
M. HALL \\ P. R. HALMOS \\ V. GANAPATHY IYER \\ R. D. JAMES
}

M. S. KNEBELMAN

I. NIVEN

T. G. OSTROM

M. M. SCHIFFER
J. J. STOKER

G. SZEKERES

F. WOLF

K. YOSIDA

\section{SUPPORTING INSTITUTIONS}

UNIVERSITY OF BRITISH COLUMBIA

CALIFORNIA INSTITUTE OF TECHNOLOGY

UNIVERSITY OF CALIFORNIA

MONTANA STATE UNIVERSITY

UNIVERSITY OF NEVADA

OREGON STATE COLLEGE

UNIVERSITY OF OREGON

UNIVERSITY OF SOUTHERN CALIFORNIA
STANFORD UNIVERSITY

UNIVERSITY OF UTAH

WASHINGTON STATE COLLEGE

UNIVERSITY OF WASHINGTON

AMERICAN MATHEMATICAL SOCIETY CALIFORNIA RESEARCH CORPORATION HUGHES AIRCRAFT COMPANY 


\section{Pacific Journal of Mathematics}

\section{Vol. 6, No. $1 \quad$ November, 1956}

David Blackwell, An analog of the minimax theorem for vector payoffs..... 1

L. W. Cohen, A non-archimedian measure in the space of real

sequences ..................................... 9

George Bernard Dantzig, Constructive proof of the Min-Max theorem ..... 25

Jim Douglas, On the numerical integration of quasilinear parabolic

differential equations ............................... 35

James Michael Gardner Fell, A note on abstract measure ............. 43

Isidore Isaac Hirschman, Jr., A note on orthogonal systems . . . . . . . . . . 47

Frank Harary, On the number of dissimilar line-subgraphs of a given

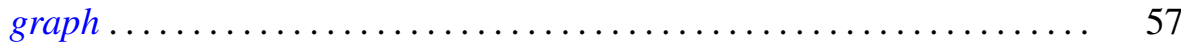

Newton Seymour Hawley, Complex bundles with Abelian group......... 65

Alan Jerome Hoffman, Morris Newman, Ernst Gabor Straus and Olga

Taussky, On the number of absolute points of a correlation ...........

Ernst Gabor Straus and Olga Taussky, Remark on the preceding paper.

Algebraic equations satisfied by roots of natural numbers . . ........ 97

Ralph D. James, Summable trigonometric series ................. 99

Gerald R. Mac Lane, Limits of rational functions . . . . . . . . . . . . . . . 111

F. Oberhettinger, Note on the Lerch zeta function ................. 117

Gerald C. Preston, On locally compact totally disconnected Abelian groups and their character groups ........................... 121

Vikramaditya Singh and W. J. Thron, On the number of singular points, located on the unit circle, of certain functions represented by

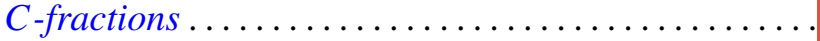

Sherman K. Stein, The symmetry function in a convex body ... 145 Edwin Weiss, Boundedness in topological rings.............

Albert Leon Whiteman, A sum connected with the series for the partition

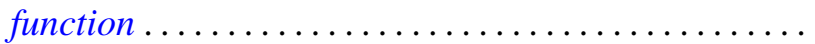

Alfred B. Willcox, Some structure theorems for a class of Banach algebras.

Joseph Lawrence Zemmer, Some remarks on p-rings and their Boolean geometry... 